\title{
Beaming Due to Comptonisation in X-ray Pulsars
}

\author{
D.K. Galloway ${ }^{1,2}$ and K. Wu ${ }^{2}$ \\ 1 School of Mathematics and Physics, University of Tasmania, GPO \\ Box 252-21, Hobart, Tasmania 7001, Australia \\ 2 Research Centre for Theoretical Astrophysics, University of Sydney, \\ Camperdown, NSW 2006, Australia
}

\begin{abstract}
Comptonisation may play an important role in determining beam patterns and hence pulse profiles in $\mathrm{X}$-ray pulsars. We describe a Monte Carlo simulation used to investigate the spectra and pulse profiles emitted by two semi-infinite homogeneous cyclindrical accretion columns diametrically located on the surface of a 'canonical' neutron star.
\end{abstract}

To date essentially all the approaches which have been used to model the emission region in $\mathrm{X}$-ray pulsars have limitations. Two phenomena in particular present difficulties for most models: the pulse profile asymmetry observed in many pulsars (see e.g. White, Swank and Holt 1983), and the sharp primary minima seen in several pulsars including GX 1+4 (Giles et al. 1999), A 0535+262 (Cemeljic \& Bulik 1998), and RX J0812.4-3114 (Reig \& Roche 1999)

We have carried out Monte Carlo simulations of the beamed emission from X-ray pulsars (Figure 1a). The algorithms of Pozdnyakov, Sobol' \& Syunyaev (1983) are used to draw the photon energy and direction, electron energies, and to calculate the fully relativistic (non-magnetic) cross-section for Compton scattering. On escaping the accretion column, general relativistic corrections including the redshift and bending of photon trajectories are calculated assuming a Schwarzschild metric. We simulate a single column for both poles of the star, and generate pulse profiles for a range of geometries simultaneously.

The model-predicted pulse profiles are in general quite symmetric, and exhibit a sharp primary minima over a range of $i$ and $\beta$. We suggest that a latitudinal asymmetry in accretion column shape and/or density may account for the observed asymmetry in pulsar profiles.

\section{References}

Cemeljic M., Bulik T. 1998, AcA, 48, 65

Giles A.B., Galloway D.K., Greenhill J.G., Storey M.C., Wilson C.A., 1999, accepted for publication by ApJ

Pozdnyakov L.A., Sobol' I.M., Syunyaev R.A. 1983, Astrophys. Space Phys. Rev., 2, 189

Reig P., Roche P. 1999, MNRAS, 306, 95

White N.E., Swank J.H., Holt S.S. 1983, ApJ, 270, 711 
a)

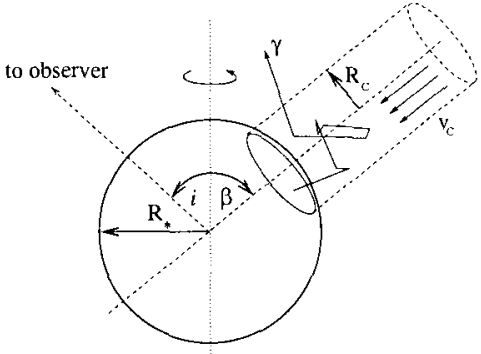

b)

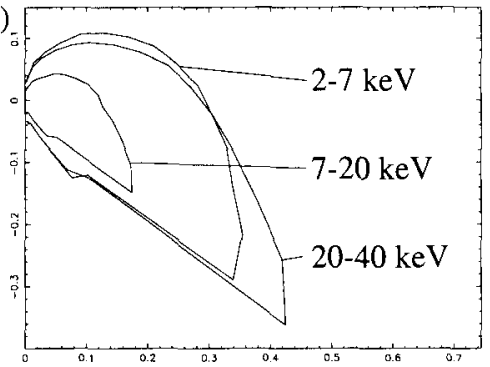

c)

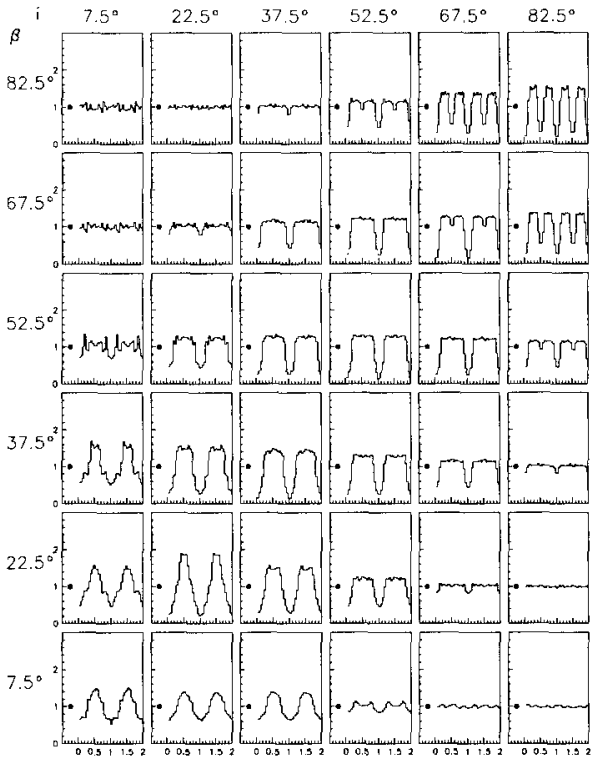

Figure 1. a) Model geometry (only one column shown for clarity). The magnetic axis of the star is aligned relative to the rotational axis by an angle $\beta$, with $i$ the inclination angle of the system with respect to the observer. Photons are emitted isotropically from each (circular) polar cap, and are then Compton scattered by the column plasma before escaping towards the observer. The plasma is flowing towards the pole with speed $v_{C}$. The temperature of the polar cap $T_{0}$, temperature $T_{e}$ and optical depth $\tau$ of the column plasma, and column diameter $R_{C}$ are all free parameters in the model. Note that $\tau=\sigma_{\mathrm{T}} R_{C} N_{e}$ where $N_{e}$ is the electron density in the column; that is, $\tau$ corresponds to photon paths from the centre of the column radially outwards. Clearly the optical depth experienced by individual photons will depend on the trajectory, and in particular with the angle relative to the column axis. b) X-ray beam pattern within several energy bands. The model parameters are $k T_{0}=1 \mathrm{keV}, k T_{e}=8 \mathrm{keV}, \tau=3, R_{C}=1 \mathrm{~km}$, and $v_{C}=0.5 c$; these conditions are such as to approximately correspond to those expected for a low-luminosity X-ray pulsar. The origin corresponds to the base of the accretion column, which is aligned along the (positive) $y$-axis. The normalisation is arbitrary.

c) Predicted pulse profiles using the same model parameters over a range of geometries. Each profile shown corresponds to a particular choice of $i$ and $\beta$, which vary between $7.5^{\circ}$ and $82.5^{\circ}$ along the $x$ - and $y$-axes respectively. Profiles are normalised to the mean and plotted over two cycles; a typical error bar is shown at the left of each panel. 